\title{
Microbial 2-butanol production with Lactobacillus diolivorans
}

\author{
Hannes Russmayer ${ }^{1,2}$, Hans Marx $^{1,2^{*}}$ (1) and Michael Sauer ${ }^{1,2,3}$ (])
}

\begin{abstract}
Background: Biobutanol has great potential as biofuel of the future. However, only a few organisms have the natural ability to produce butanol. Amongst them, Clostridium spp. are the most efficient producers. The high toxicity of biobutanol constitutes one of the bottlenecks within the biobutanol production process which often suffers from low final butanol concentrations and yields. Butanol tolerance is a key driver for process optimisation and, therefore, in the search for alternative butanol production hosts. Many Lactobacillus species show a remarkable tolerance to solvents and some Lactobacillus spp. are known to naturally produce 2-butanol from meso-2,3-butanediol (meso-2,3-BTD) during anaerobic sugar fermentations. Lactobacillus diolivorans showed already to be highly efficient in the production of other bulk chemicals using a simple two-step metabolic pathway. Exactly, the same pathway enables this cell factory for 2-butanol production.

Results: Due to the inability of L. diolivorans to produce meso-2,3-BTD, a two-step cultivation processes with Serratia marcescens has been developed. S. marcescens is a very efficient producer of meso-2,3-BTD from glucose. The process yielded a butanol concentration of $10 \mathrm{~g} / \mathrm{L}$ relying on wild-type bacterial strains. A further improvement of the maximum butanol titer was achieved using an engineered L. diolivorans strain overexpressing the endogenous alcohol dehydrogenase pduQ. The two-step cultivation process based on the engineered strain led to a maximum 2-butanol titer of $13.4 \mathrm{~g} / \mathrm{L}$, which is an increase of $34 \%$.

Conclusion: In this study, L. diolivorans is for the first time described as a good natural producer for 2-butanol from meso-2,3-butanediol. Through the application of a two-step cultivation process with S. marcescens, 2-butanol can be produced from glucose in a one-vessel, two-step microbial process.
\end{abstract}

Keywords: Industrial microbiology, Metabolic engineering, 2,3-Butanediol, 2-Butanone, Serratia marcescens

\section{Background}

Fossil oil constitutes the primary energy carrier worldwide, whereof around $80 \%$ are consumed within the transport sector. Environmental concerns and limited resources stimulate the quest for renewable alternatives for fuel production. Biodiesel and bioethanol are the most frequently used biofuels for diesel and gasoline engines today. In the last decade, biobutanol is becoming more and more important as alternative to the commonly used biofuels. Several advantages of biobutanol,

\footnotetext{
*Correspondence: hans.marx@boku.ac.at

2 Department of Biotechnology, Institute of Microbiology and Microbial Biotechnology, BOKU-VIBT University of Natural Resources and Life

Sciences, Vienna, Muthgasse 18, 1190 Vienna, Austria

Full list of author information is available at the end of the article
}

such as a higher energy content, usability in pure form or the ability to blend it in every concentration with gasoline, favour butanol over other biofuels [1]. Butanol is a C4-alcohol. Four different isomers exist, of which 1-butanol, 2-butanol and isobutanol are the most promising candidates for biofuel production.

The largest share of the global biobutanol market has 1-butanol. Traditionally, 1-butanol is produced by ABE (acetone-butanol-ethanol) fermentation of solventogenic Clostridia, mainly C. acetobutylicum and C. beijerinckii. As 1-butanol is a toxic metabolite, the titers and yields are rather low. Usually, titers for wild-type strains on glucose as carbon source are around $15-20 \mathrm{~g} / \mathrm{L}$ and yields are around $0.20 \mathrm{~g} / \mathrm{g}$ [2]. The high toxicity of butanol constitutes a major bottleneck for high-level production 
of butanol with Clostridium spp. Metabolic engineering of $C$. acetobutylicum to increase 1-butanol production focused mainly on improving butanol tolerance and avoiding by-product formation [2]. One of the highest titers for metabolically engineered $C$. acetobutylicum strains was around $20 \mathrm{~g} / \mathrm{L}[3,4]$. Only via process engineering, in situ removing of 1-butanol from the broth by gas stripping or liquid-liquid extraction, titers could be further improved. For a 1-butanol fermentation process using a long-term-adapted mutant of $C$. acetobutylicum JB200 in combination with in situ removal of 1-butanol, a final titer of $118 \mathrm{~g} / \mathrm{L}$ for the overall fermentation was reached [5].

Isobutanol is also a promising candidate for biobutanol production, because of its lower toxicity for microbial cells. On academic level, isobutanol is mainly produced by metabolically engineered Escherichia coli and Saccharomyces cerevisiae via introduction of genes of the keto acid pathway. Engineered E. coli strains reached up to $22 \mathrm{~g} / \mathrm{L}$ and further process engineering led to $50 \mathrm{~g} / \mathrm{L}$ of isobutanol [6]. Several companies, such as Gevo Inc. (http://www.gevo.com) are involved in scale-up of the fermentation process to an industrial level.
Up to now, the third isomer 2-butanol lives in the shadow of the other two isomers, but is with its comparable higher octane number and lower toxicity to 1-butanol of considerable interest for the biofuel industry.

2-Butanol is known to be produced by some Lactobacillus spp. through reduction of 2,3-butanediol (2,3-BTD) during anaerobic sugar fermentation. Two consecutive enzymatic steps carry out this reduction. The first step is the dehydration of meso-2,3-BTD to 2-butanone by a vitamin $\mathrm{B}_{12}$-dependent glycerol dehydratase. In the second step, 2-butanone is reduced to 2-butanol by an alcohol dehydrogenase, which accepts secondary alcohols. Both enzymatic steps are located within bacterial microcompartments (BMC), organelle-like proteinaceous structures.

Typically, BMCs found in Lactobacillus spp. are assigned to the group of propanediol utilisation (pdu) micro-compartments, due to their natural metabolic function for degradation of 1,2-propanediol. Interestingly, the same metabolic pathway located in the pdu micro-compartment is able to convert different substrates with similar chemical structures, bearing at least one vicinal diol (Fig. 1).

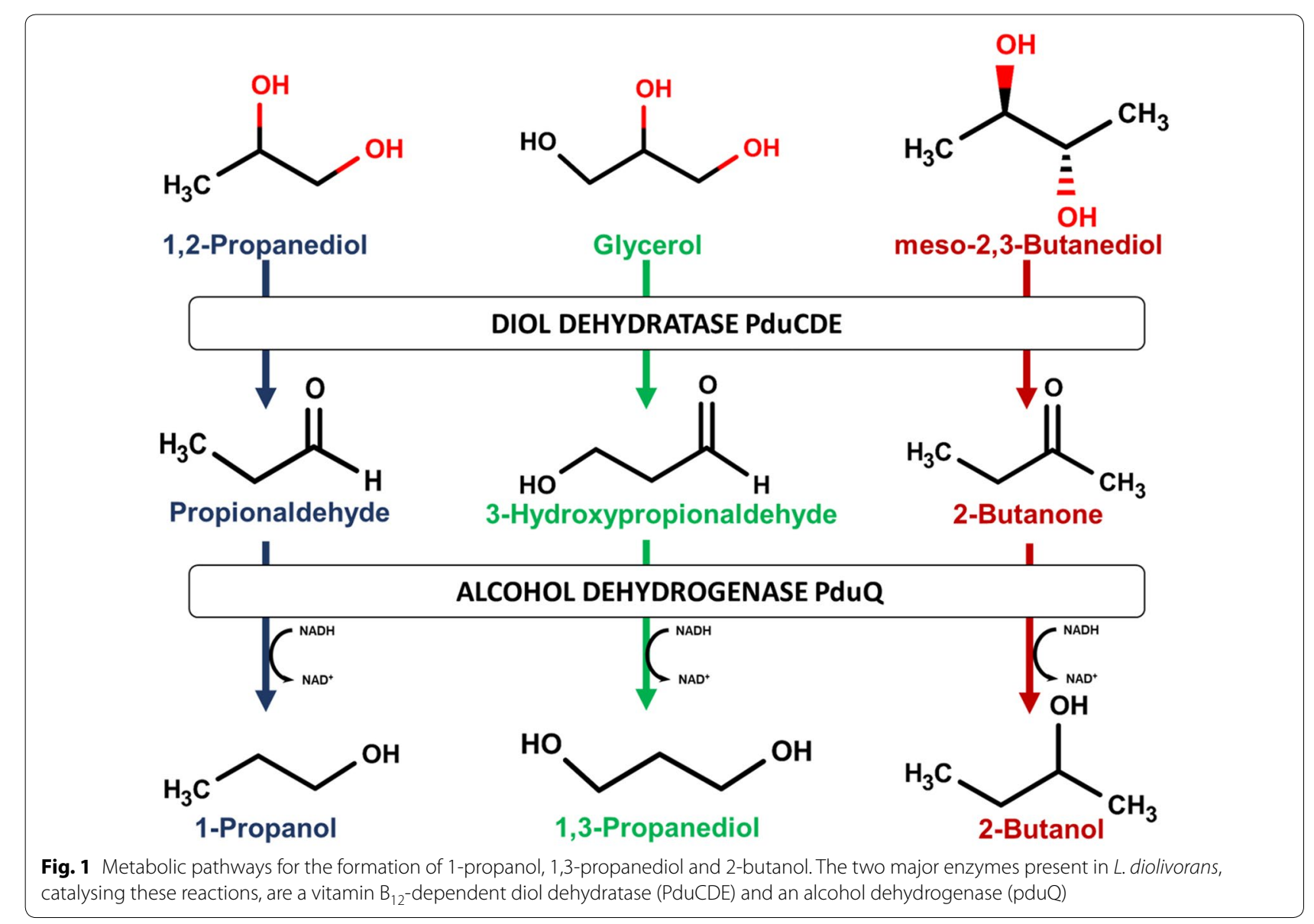


A Lactobacillus spp. known to use this metabolic pathway in a very efficient way is Lactobacillus diolivorans. L. diolivorans is able to produce up to $92 \mathrm{~g} / \mathrm{L}$ of 1,3-propanediol (1,3-PDO) in fed-batch cultivations [7]. Additionally, this cell factory is able to produce up to $35 \mathrm{~g} / \mathrm{L}$ of 3 -hydroxypropionaldehyde (3-HPA), the intermediate product of the metabolic pathway (Fig. 1). Having this efficient and metabolically well-balanced pathway, $L$. diolivorans is also a potential candidate for 2-butanol production from 2,3-BTD. 2,3-BTD has two stereoactive centres and therefore, three different isomers are found in nature, which are the optical active $\mathrm{R}$ - and $\mathrm{S}$-form and the inactive meso-form. All three isomers are produced by microbial strains found in nature. However, the ratio between the different isomers varies amongst the bacteria capable of producing 2,3-BTD [8]. 2,3-BTD is usually formed via the pyruvate-diacetyl/acetoin pathway. This pathway is found in lactic acid bacteria and glucose and citrate fermenting microorganisms, belonging to the genus Klebsiella, Enterobacter, Bacillus, and Serratia [9]. The mentioned pathway consists of 3 enzymatic steps starting by the condensation of two pyruvate molecules to alpha-acetolactate with the help of alpha-acetolactate synthase. In the next step, alpha-acetolactate decarboxylase decarboxylates alpha-acetolactate to acetoin. Acetoin is then used as a precursor for the production of 2,3-BTD catalysed by different acetoin reductases (or 2,3-BTD dehydrogenases). The ratio between the different isoforms for 2,3-BTD is dependent on the expressed dehydrogenase enzymes.

A variety of lactic acid bacteria (such as Lactococcus lactis, Lactobacillus plantarum and L. brevis) are able to produce 2,3-BTD. However, the 2,3-BTD titers obtained are very low and a racemic mixture of 2,3-BTD is produced. For high-level production of 2-butanol, a higher titer of meso-2,3-BTD and a higher stereospecificity for the meso-form are needed, because known dehydratase enzymes are usually specific for one racemic form. The benchmark for 2,3-BTD production is Klebsiella pneumoniae and Serratia marcescens reaching titers up $75.2 \mathrm{~g} / \mathrm{L}$ in fed-batch cultivations with glucose as carbon source [10]. Additionally, S. marcescens has the advantage of producing only meso-2,3-BTD, which seems ideal for the production of 2-butanol using $L$. diolivorans $[9,11]$.

In this study, we investigated the potential of $L$. diolivorans for 2-butanol production. This lactic acid bacterium has several characteristics, which make this organism an interesting production host. Lactic acid bacteria are generally known to have a high tolerance to several stress conditions, such as high concentrations of acids or alcohols [12]. For example, lactic acid bacteria tolerate up to $3 \%$ butanol in the cultivation medium; whereas, Clostridium spp., the benchmark for 1-butanol production, typically only tolerate around $2 \%$.

Furthermore, this organism already proved to be an efficient cell factory for metabolite production [7, 13]. The meso-2,3-BTD production by lactic acid bacteria is not high enough to realise the full potential for 2-butanol production of $L$. diolivorans. Therefore, a two-step co-cultivation process with $S$. marcescens in batch mode was developed. S. marcescens was selected for production of meso-2,3-BTD from glucose $[9,11]$. In the first step of the process, $S$. marcescens was used to produce stereo specifically meso-2,3-BTD from glucose followed by heat inactivation of $S$. marcescens. The accumulated meso-2,3-BTD is then converted during anaerobic fermentation with glucose into 2-butanol by L. diolivorans.

\section{Results}

\section{Butanol tolerance of $L$. diolivorans}

Butanol is highly toxic for most microorganisms also at low concentrations $(<15 \mathrm{~g} / \mathrm{L}$ for 1 -butanol). Increasing concentrations of butanol affect the membrane fluidity and impair membrane transport functions, which leads to uncontrolled efflux of intracellular components, such as proteins and metabolites [14]. Lactic acid bacteria (such as L. brevis) already showed to have a higher butanol tolerance than most other organism $[12,15]$. Therefore, it is of interest to determine the butanol tolerance of $L$. diolivorans. The tested $L$. diolivorans wild-type strain showed a butanol tolerance up to $25 \mathrm{~g} / \mathrm{L}$ 2-butanol, where it still reached $88 \%$ of the maximum $\mathrm{OD}_{600}$ after $72 \mathrm{~h}$ of incubation (Fig. 2). The maximum $\mathrm{OD}_{600}$ refers to the optical density reached in the control media, where no 2-butanol was added (MRS medium $+0 \%$ 2-butanol). A drastic decrease in growth was observed at $30 \mathrm{~g} / \mathrm{L}$ 2-butanol, where only $30 \%$ of the maximum $\mathrm{OD}_{600}$ was reached, but the (non-adapted) organisms were still able to grow. The obtained results show that $L$. diolivorans has a high tolerance to solvents and is an ideal microbial cell factory for 2-butanol production. Furthermore, it was shown that Lactobacillus spp. have a comparable tolerance to the more toxic 1-butanol than Clostridium strains, which are the benchmark for butanol production. Wild-type Clostridium spp. (such as C. acetobutylicum) usually tolerate butanol concentrations up to $20 \mathrm{~g} / \mathrm{L}$ [15, 16]. Only engineered or mutagenized Clostridium strains can tolerate a bit higher concentrations of butanol. Other organisms engineered for butanol production (Escherichia coli, Saccharomyces cerevisiae) exhibit mostly a lower butanol tolerance than Clostridium spp. 


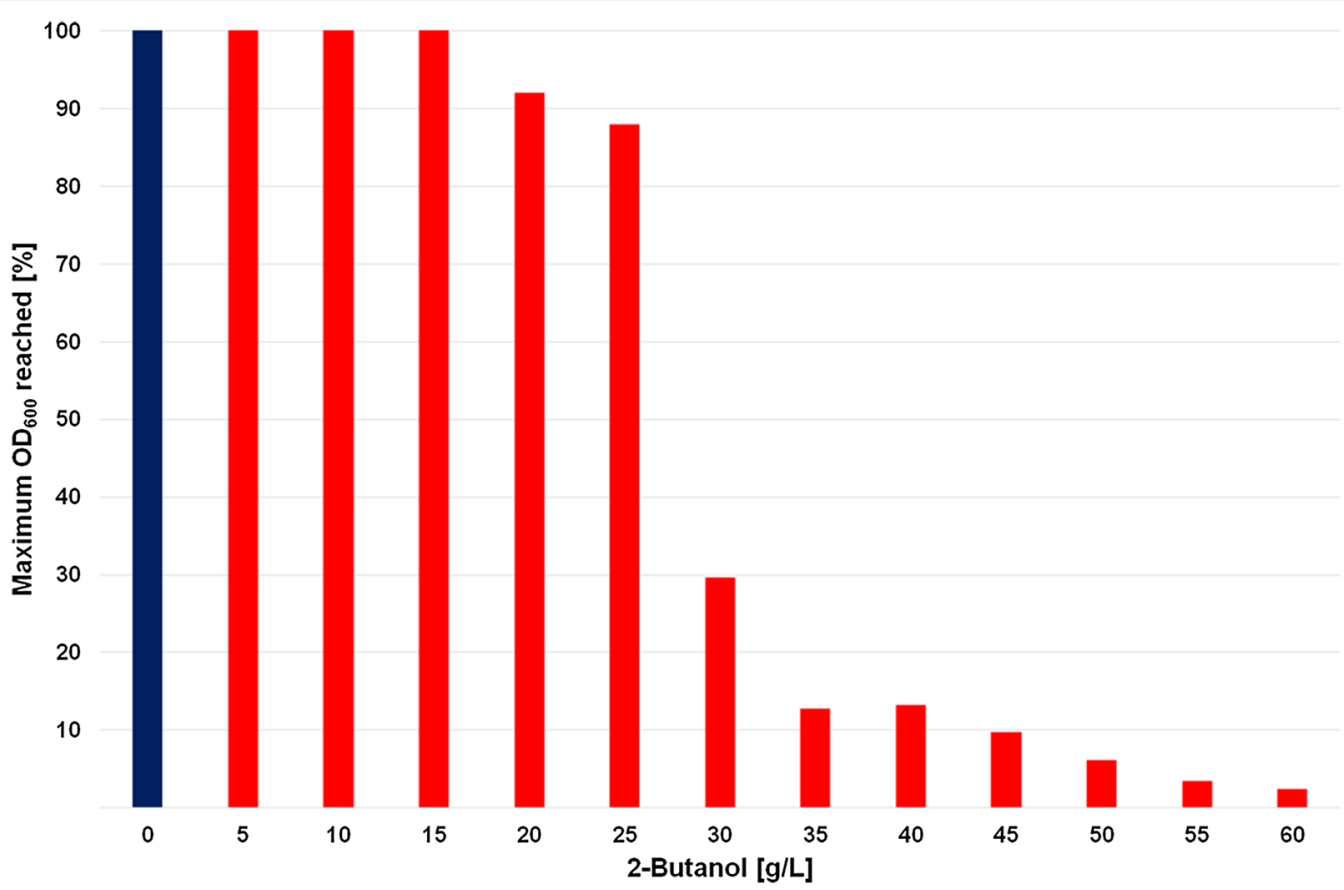

Fig. 2 Maximum $\mathrm{OD}_{600}$ of $\mathrm{L}$. diolivorans on medium with increasing butanol concentrations relative to medium without butanol

\section{Microbial meso-2,3-butanediol production with $S$. marcescens}

Glycerol dehydratases found in lactic acid bacteria are highly stereospecific. Some have been described to only convert the meso-form of 2,3-BTD further into 2-butanone [17]. To determine if the glycerol dehydratase of $L$. diolivorans shows the same specificity, anaerobic batch cultivations with a racemic mixture of 2,3-BTD were performed. GC-MS analysis of the supernatant showed that during the anaerobic batch fermentation exclusively the meso-form of 2,3-BTD was converted into 2-butanol and the other two isomers (R-, S-form) remained untouched (data not shown). Therefore, it became clear that a process for 2,3-BTD should also exclusively yield the meso-form to aim for high overall efficiency.

Our efforts concentrated on the enantiospecific production of meso-2,3-BTD from glucose with $S$. marcescens. To evaluate the potential of S. marcescens DSMZ 14187 for meso-2,3-BTD production, batch cultivations with glucose as sole carbon source were conducted (Fig. 3). For all performed cultivations, the highest titers of meso-2,3-butanediol were reached at around $19 \mathrm{~h}$ with $35-39.4 \mathrm{~g} / \mathrm{L}$ and a conversion yield between 0.38 and $0.44 \mathrm{~g} / \mathrm{g}$, which is close to the theoretical maximum of $0.50 \mathrm{~g} / \mathrm{g}$ (Fig. 3). Our results correlate well with reported batch cultivations reaching a final titer of $42.5 \mathrm{~g} / \mathrm{L}$ [18], showing S. marcescens DSMZ 14187 as a robust and very efficient producer of meso-2,3-BTD. Further, HPLC analysis showed that after glucose depletion, the produced meso-2,3-BTD was degraded most likely to its precursor acetoin. Therefore, it is of high importance to stop the fermentation process before glucose depletion to reach the maximum titer of meso-2,3-BTD. A part of meso2,3-BTD the byproducts lactate, ethanol and $\mathrm{CO}_{2}$ were observed during the batch process on glucose. Acetate was co-utilised by $S$. marcescens as carbon source.

\section{Production of 2-butanol from meso-2,3-butanediol with $L$. diolivorans}

Serratia marcescens was heat inactivated, when the maximum titre of around $39 \mathrm{~g} / \mathrm{L}$ of meso-2,3-BTD was reached (Fig. 4). Heat inactivation was essential to prevent $S$. marcescens to further convert glucose, which is required as carbon source for the added lactic acid bacteria in the second step of the cultivation. To allow the proper growth of $L$. diolivorans, 5 times concentrated MRS plus glucose was added to the bioreactor containing the heat inactivated first-step culture. The concentration for glucose and meso-2,3-BTD at the beginning of the second phase were both $30 \mathrm{~g} / \mathrm{L}$. L. diolivorans, as a heterofermentative lactic acid bacterium, metabolises glucose to its typical fermentation products lactic acid, ethanol and $\mathrm{CO}_{2}$. In addition, acetate, 2-butanone and 2-butanol accumulated during the batch phase (Table 1). Acetate is a product accumulated on glucose when further electron 


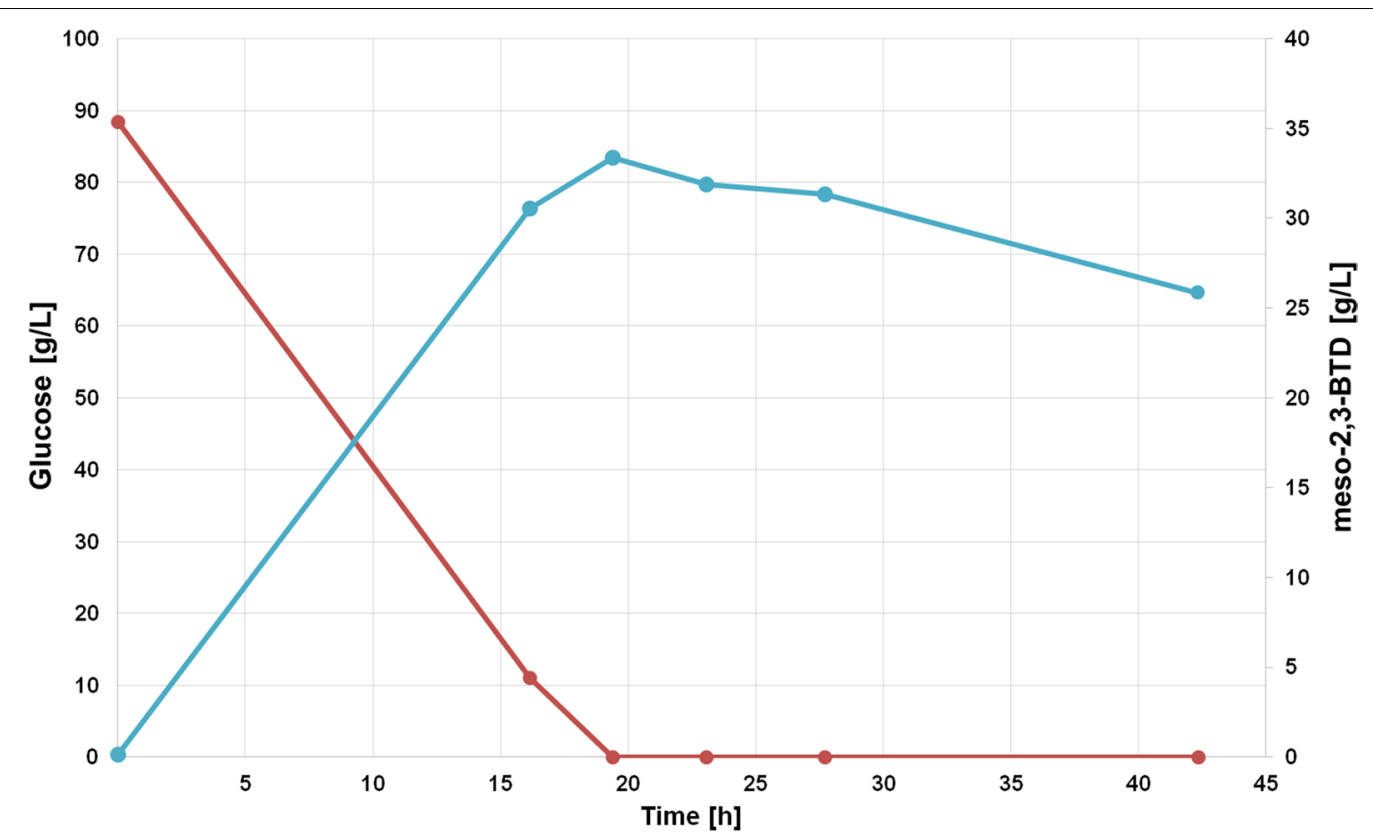

Fig. 3 Batch cultivation of S. marcescens on glucose for meso-2,3-butanediol production. Glucose, closed red circles; meso-2,3-BTD, closed turquoise circles

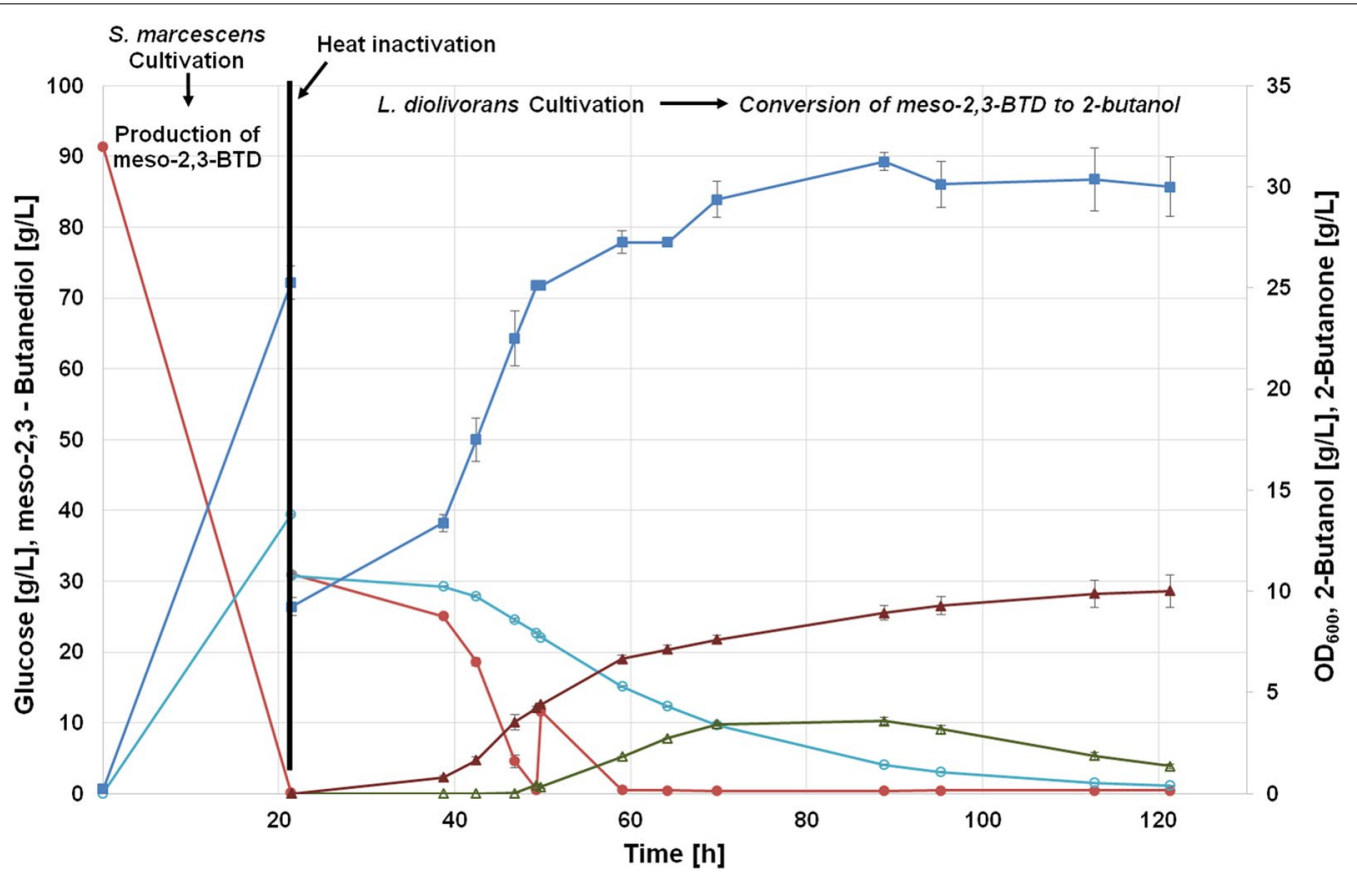

Fig. 4 Two-step cultivation process in batch mode with wild-type L. diolivorans. Glucose, closed red circles; meso-2,3-BTD, open turquoise circles; $\mathrm{OD}_{600}$, closed blue squares; 2-butanone, opened green triangle; 2-butanol, closed brown triangle. Error bars represent the standard deviation of three independent replicates

acceptors such as glycerol or 2,3-BTD as in this case are present [13]. The maximum 2-butanol titre reached was $10.0 \mathrm{~g} / \mathrm{L}$ after $121 \mathrm{~h}$, which is the highest titer reported for microbial 2-butanol production (Fig. 4). Comparable initial concentration of glucose and glycerol, instead of meso-2,3-BTD, yielded $23.8 \mathrm{~g} / \mathrm{L}$ of 1,3-PDO after $147 \mathrm{~h}$ 
Table 1 Summary of fermentation productions after $120 \mathrm{~h}$ of fermentation

\begin{tabular}{llllllll}
\hline & Glucose (g/L) & Lactate $(\mathbf{g} / \mathbf{L})$ & Acetate $(\mathbf{g} / \mathbf{L})$ & $\begin{array}{l}\text { meso23-BTD } \\
(\mathbf{g} / \mathbf{L})\end{array}$ & Ethanol (g/L) & $\begin{array}{l}\text { 2-Butanone } \\
(\mathbf{g} / \mathbf{L})\end{array}$ & 2-Butanol (g/L) \\
\hline $\begin{array}{l}\text { Wild type } \\
\begin{array}{l}\text { pduQ overexpres- } \\
\text { sion strain }\end{array}\end{array}$ & 0.00 & 19.87 & 19.04 & 1.12 & 9.96 & 1.35 & 10.01 \\
\hline
\end{tabular}

[13]. Interestingly, looking at the final product titers and yields, the efficiency of the metabolic pathway for reduction of either meso-2,3-BTD or glycerol to their respective products is different. During glucose/meso2,3-BTD fermentation, the accumulation of the intermediate product 2-butanone is observed. The formation of 2-butanone started at around $50 \mathrm{~h}$ of cultivation and reached the highest concentration with $3.6 \mathrm{~g} / \mathrm{L}$ after $88 \mathrm{~h}$ (Fig. 4). However, the produced 2-butanone was depleted at the end of the fermentation. 2-butanone was either converted to 2-butanol or evaporated from the fermentation broth due to nitrogen gassing of the reactor. The accumulation of 2-butanone points to a bottleneck at the second step of the metabolic pathway, which is catalysed by an alcohol dehydrogenase. For glucose/glycerol co-fermentations, no accumulation of the intermediate 3-HPA was observed, showing that this pathway is well balanced for glycerol conversion [19]. 2-butanone accumulation can be explained by the fact that reduction to 2-butanol requires a secondary alcohol dehydrogenase. We speculate that the natural alcohol dehydrogenase is very efficient for primary alcohols but less efficient for secondary alcohols.

More glucose was added to the reactor at the batch end (around $28 \mathrm{~h}$ ) to ensure sufficient supply with NADH to fully convert meso-2,3-BTD to 2-butanol. The glucose pulse was $10 \mathrm{ml}$ of a $50 \%$ glucose solution and final glucose concentration after the pulse was $11 \mathrm{~g} / \mathrm{L}$ (Fig. 4). The conversion yield for 2-butanol from meso-2,3-BTD was $0,37 \mathrm{~g} / \mathrm{g}$ and the overall yield for production of 2-butanol on glucose was $0.06 \mathrm{~g} / \mathrm{g}$.

\section{Overexpression of the endogenous 1,3-propanediol} oxidoreductase (pduQ) increases 2-butanol formation

Batch cultivations of wild-type $L$. diolivorans indicated that the bottleneck for 2-butanol production is the reduction of 2-butanone to 2-butanol. To enhance this conversion, the overexpression of the endogenous 1,3-propanediol oxidoreductase (pduQ) was our first choice. Its promiscuous activity leads to the formation of 2-butanol from 2-butanone, as observed in the wild-type strain. The $L$. diolivorans strain overexpressing pduQ was cultivated with $S$. marcescens following the same process design (Fig. 5). The pduQ overexpression strain showed already in a previous study an increased 1,3-propanediol production from glycerol in batch cultivations, which indicates that overexpressing of pduQ positively influences product formation. Furthermore, the authors showed that pduQ enzyme activity was twice as high as in the wild-type strain [20].

Using the overexpression strain, the final 2-butanol titer was increased by $34 \%$ reaching a maximum of $13.4 \mathrm{~g} / \mathrm{L}$ after $112 \mathrm{~h}$ of cultivation (Fig. 5). Furthermore, the overall yield on glucose increased to $0.10 \mathrm{~g} / \mathrm{g}$. Interestingly, the formation of 2-butanone was still observed and reached still a maximum of around $3.5 \mathrm{~g} / \mathrm{L}$ which is very similar to the wild-type strain (Fig. 6). However, conversion of 2-butanone was faster and more efficient in the pduQ overexpression strain, as 2-butanone was used up after $121 \mathrm{~h}$ (Fig. 6). At the same time point, still $0.3 \mathrm{~g} / \mathrm{L}$ of 2-butanone was found in the fermentation broth of the wild-type strain. Interestingly, a slightly lower concentration of lactate and an increased acetate concentration were observed (Table 1). This may indicate that additional NADH was created by lactate degradation and further used for reduction of meso-2,3-BTD reduction to 2-butanol. The final ethanol concentrations were the same for the engineered and wild-type strain. Overall, the final 2-butanol titer and conversion rate were increased by the overexpression of pduQ.

\section{Discussion}

So far, no efficient microbial production process for 2-butanol has been described. In this study, we propose L. diolivorans as a promising production organism, accumulating a 2-butanol concentration of $13.4 \mathrm{~g} / \mathrm{L}$.

The high toxicity of butanol is a general problem during the production process. Therefore, tolerance to butanol is an important point to consider for the selection of a microbial cell factory for this compound. Only few data are available about butanol tolerance of other organisms than Clostridium spp. However, some reports showed that Lactobacillus spp. have a high tolerance to solvents and acids [12, 15, 22], which makes them interesting hosts for such compounds. Smallscale experiments on MRS + Glucose with increasing 2-butanol concentrations showed that wild-type $L$. diolivorans tolerates 2-butanol up to concentrations of about $30 \mathrm{~g} / \mathrm{L}$. This is in good accordance with obtained data for butanol tolerance of other Lactobacillus spp. 


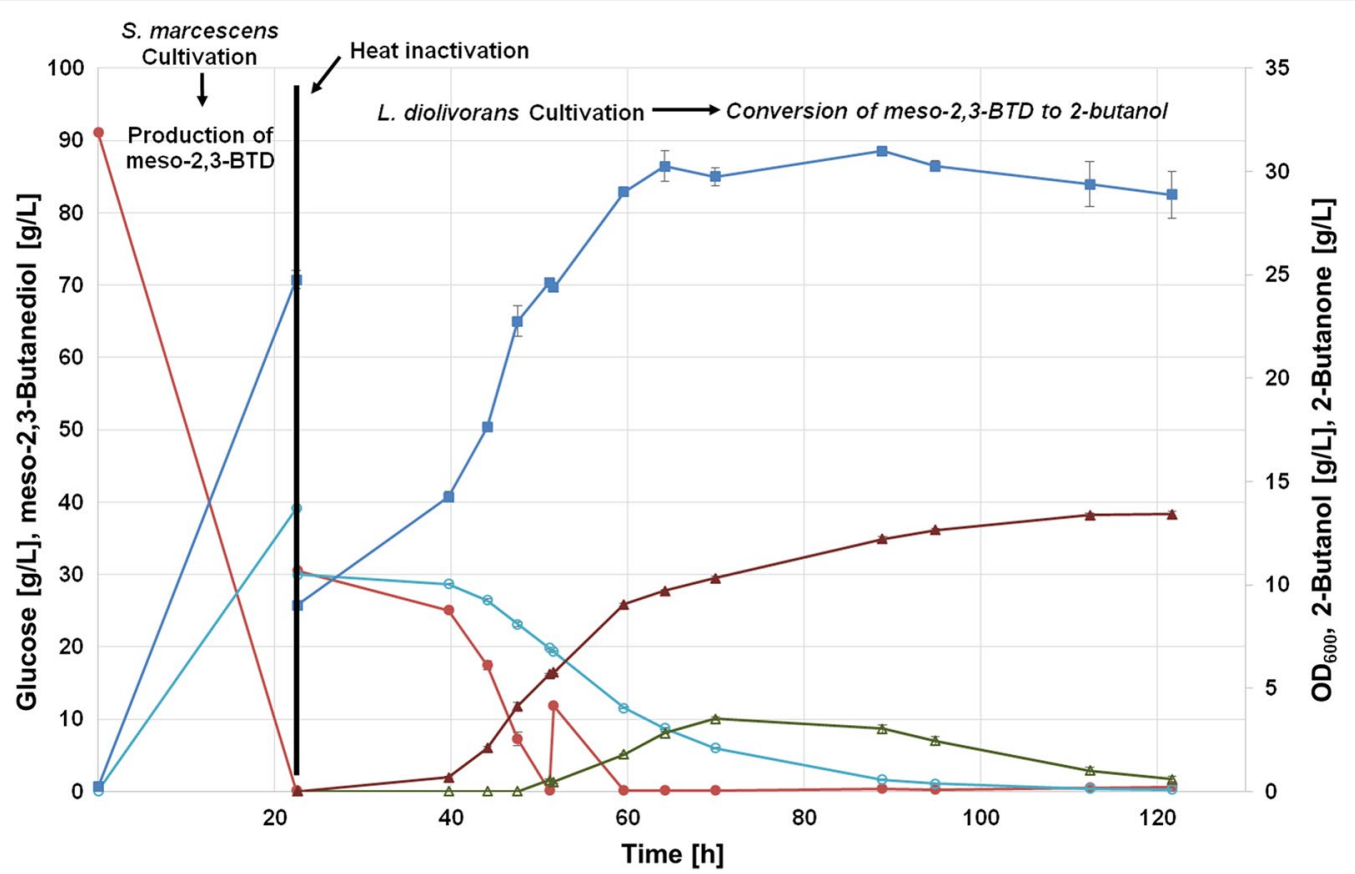

Fig. 5 Two-step cultivation process in batch mode with L. diolivorans overexpressing pduQ. Glucose, closed red circles; meso-2,3-BTD, open turquoise circles; $\mathrm{OD}_{600}$, closed blue squares; 2-butanone, opened green triangle; 2-butanol, closed brown triangle. Error bars represent the standard deviation of three independent replicates

[15, 22, 23]. Interestingly, Clostridia spp., the typical organisms used for butanol production, tolerate this solvent only until concentrations of $20 \mathrm{~g} / \mathrm{L}[2,23]$. Typical hosts for metabolic engineering, such as E. coli or S. cerevisiae already have severe growth problems at butanol concentrations of around 10-15 g/L [15]. This points to the potential of $L$. diolivorans as a cell factory for 2-butanol production.

The metabolic pathway used by $L$. diolivorans for the formation of 2-butanol is a rather simple two-step metabolic pathway. On the contrary, the production of 1-butanol with Clostridium spp. underlies complex regulatory mechanisms switching from acidogenesis to solventogenesis [2].

The aforementioned metabolic pathway is widespread among lactic acid bacteria. For example, Lactobacillus brevis was also identified to be capable of 2-butanol production. Interestingly, Speranza et al. showed that the glycerol dehydratase of $L$. brevis stereospecifically converts only the meso-form of 2,3-BTD into 2-butanone $[17,21]$. The same specificity for the meso-form of 2,3-BTD was also found for the glycerol dehydratase of $L$. diolivorans. Lactobacillus strains can produce 2,3BTD, but product titers are rather low and furthermore, a racemic mixture of all three isoforms is produced. Therefore, S. marcescens, a well-known production host known for high yield and stereospecific production of
meso-2,3-BTD from glucose, was used for the two-stage production of 2-butanol.

The achieved 2-butanol titer of $13.4 \mathrm{~g} / \mathrm{L}$ by applying the developed two-step co-cultivation process is around 30 times higher than the so far reported 2-butanol titer in literature (Table 2). In another study, 42 Lactobacillus isolates were screened for 2-butanol production from 2,3-BTD, out of which two isolates of L. brevis performed best (Table 2). The maximum titer reached was $0.8 \mathrm{~g} / \mathrm{L}$ of 2-butanol from $3 \mathrm{~g} / \mathrm{L}$ of meso-2,3-BTD after $170 \mathrm{~h}$ of cultivation. Interestingly, 2-butanol production was only observed, when $L$. brevis was cultivated on defined medium. The authors explained the observed result by repression of genes needed for conversion of meso-2,3-BTD due to cultivation on rich media, such as MRS medium [21]. The engineering of $S$. cerevisiae for 2-butanol by introducing the same two-step metabolic pathway, also used by Lactobacillus strains, led to a maximum titer of $4 \mathrm{mg} / \mathrm{L} 2$-butanol and $2 \mathrm{mg} / \mathrm{L}$ of 2 -butanone [24].

The 2-butanol titers achieved with $L$. diolivorans as production host are comparable to 1-butanol titers achieved by wild-type $C$. acetobutylicum, which are usually around $12 \mathrm{~g} / \mathrm{L}$ (Table 2 ). The strategy to improve 2-butanol titers by overexpressing the endogenous primary alcohol dehydrogenase pduQ was successful. Interestingly, 2-butanone formation was still observed 

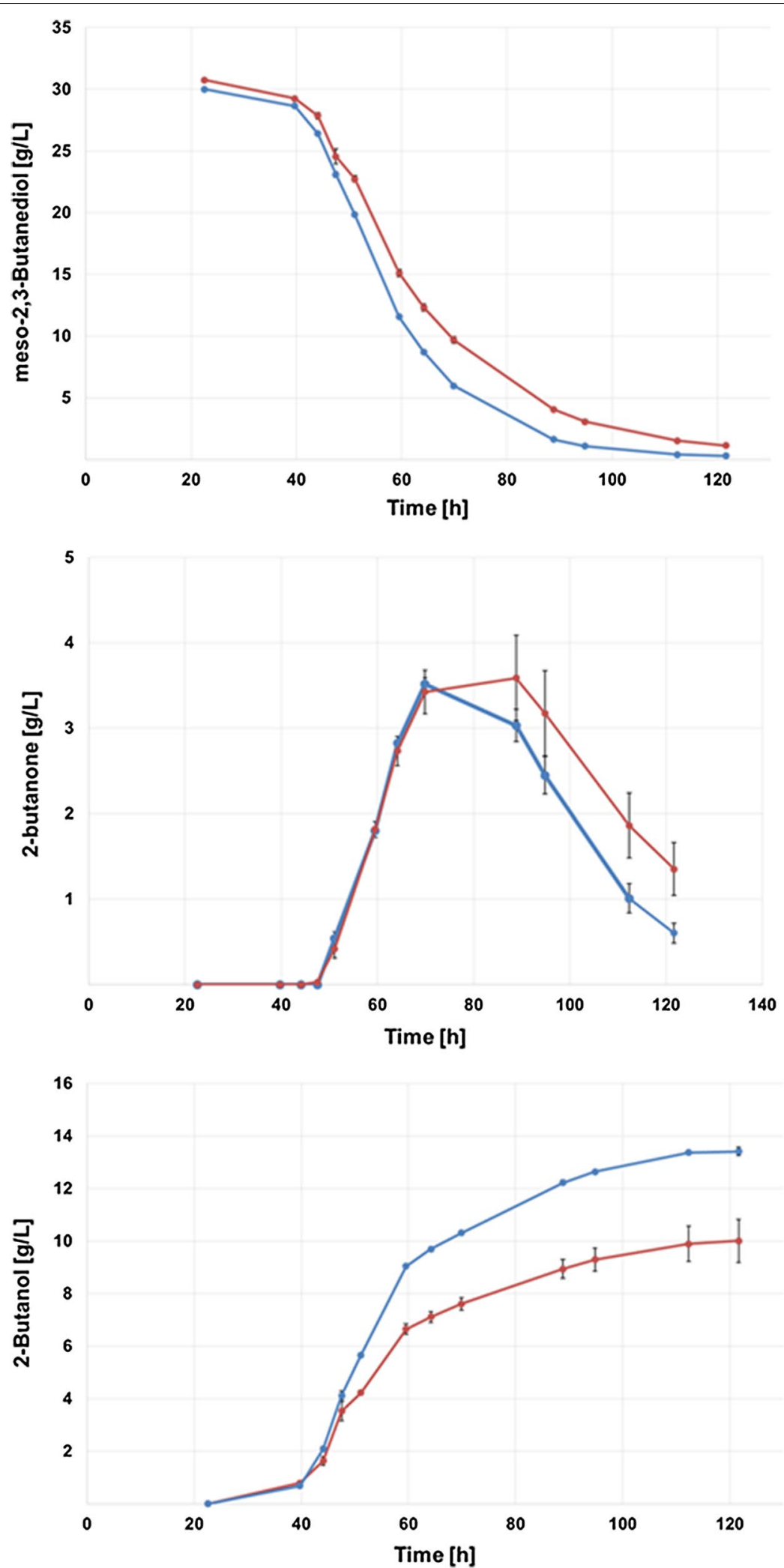

Fig. 6 Comparison of 2-butanol production for the wild-type and the pduQ overexpressing strain. Wild type, closed red circles; pduQ overexpression strain, closed turquoise circles. Error bars represent the standard deviation of three independent replicates 
Table 2 Summary of butanol producing strains

\begin{tabular}{lllllll}
\hline Strain & Product & wt/engin. & Genetic engineering/processes engineering & Titer (g L-1) & Yield (g/g-1) & References \\
\hline Lactobacillus diolivorans & 2-Butanol & wt & - & 10.0 & 0.06 & This study \\
Lactobacillus diolivorans & 2-Butanol & eng. & Overexpression of PduQ & 13.4 & 0.10 & This study \\
Lactobacillus brevis & 2-Butanol & wt & - & 0.88 & - & {$[21]$} \\
Lactobacillus buchneri & 2-Butanol & wt & - & 0.04 & - & {$[21]$} \\
Saccharomyces cerevisiae & 2-Butanol & eng. & Overexpression PduCDEGH and ADH & 0.01 & - & {$[24]$} \\
Clostridium acetobutylicum & 1-Butanol & wt. & - & 11.8 & 0.18 & {$[2]$} \\
Clostridium acetobutylicum & 1-Butanol & eng. & Knockout of buk and pta & 18.9 & 0.29 & {$[2]$} \\
Clostridium acetobutylicum & 1-Butanol & eng. & Adaptive mutagenesis & 20.3 & 0.23 & {$[4]$} \\
\hline
\end{tabular}

wt wild type, eng. engineered strain

and around $3.7 \mathrm{~g} / \mathrm{L}$ was reached, which is the same concentration also reached for the wild-type strain. In both cases, the accumulated 2-butanone vanished until the end of the fermentation.

A reason for the observed phenomenon can be that pduQ is a primary alcohol dehydrogenase, whereas the reduction of 2-butanone to 2-butanol requires a secondary alcohol dehydrogenase. Therefore, kinetics of the 2-butanone reduction is rather slow as this is not the favoured substrate of $\mathrm{pduQ}$, but overexpression of pduQ still leads to an increase in 2-butanol titers. An approach for further increasing the efficiency of 2-butanol formation will be the overexpression of a heterologous secondary alcohol dehydrogenase. For $S$. cerevisiae, it has been already shown that the overexpression of a secondary alcohol dehydrogenase in combination with the postulated pathway (Fig. 1) allows efficient 2-butanol production.

The theoretical yield for the conversion of 2-butanol from meso-2,3-BTD is $0.82 \mathrm{~g} / \mathrm{g}$. For the performed co-cultivation of the wild-type strain, we only reached $0.37 \mathrm{~g} / \mathrm{g}$ and for the pduQ overexpression strain $0.49 \mathrm{~g} / \mathrm{g}$. The difference between the theoretical and observed yield was unexpected, as there are no side products of 2,3-BTD conversion known. Furthermore, we do not find any unidentified peaks in the chromatograms of the supernatants. Therefore, only the degradation of the product or loss of substrate and/or product via the gas phase explains the observed difference in yield. 2-butanone and 2-butanol are volatile substances and evaporation from the fermentation broth is a major problem in industrial processes. It is, therefore, very likely that the constant nitrogen gassing, assuring anaerobic conditions throughout the entire cultivation, causes such an evaporation of 2-butanol and/or 2-butanone and explains the lower observed product yield from meso-2,3-BTD. 2-butanol titers and yields could be further increased by avoiding nitrogen gassing or the implementation of a more efficient cooling trap for the off gas than we actually have. Another point to consider for designing an economically efficient process is the optimization of the cultivation medium. In this study, all performed cultivation steps were done with complex MRS medium to avoid nutrient limitation and allow the best possible conditions for the conversion of meso-2,3-BTD into 2-butanol by L. diolivorans. The complex components of the MRS medium, such as casein peptone, meat extract and yeast extract, contribute mainly to the high costs of the MRS medium. A goal for further media optimization can be the reduction or omitting of the complex components.

Another strategy to decrease media costs is the use of a chemically defined medium. For L. brevis, it was already shown that conversion of meso-2,3-BTD into 2-butanol is possible on such defined media [21].

\section{Conclusion}

In this study, we identified $L$. diolivorans as potential host for the production of 2-butanol from meso-2,3BTD during anaerobic glucose fermentation. Up to $10 \mathrm{~g} / \mathrm{L}$ of 2-butanol was produced by wild-type $L$. diolivorans during a two-step cultivation process with $S$. marcescens. Using an engineered strain of $L$. diolivorans, overexpressing the endogenous alcohol dehydrogenase pduQ, 2-butanol concentrations were further increased to $13.4 \mathrm{~g} / \mathrm{L}$. To our knowledge, this is the highest titre described for microbial 2-butanol production so far. The obtained 2-butanol concentrations are in the range of 1-butanol concentrations typically reached by wild-type Clostridia strains, which are considered as the best natural producers for butanol.

As $L$. diolivorans is able to tolerate higher 2-butanol concentrations, a further increase in final titers is possible. To reach higher titers, further processes engineering is needed, as evaporation of 2-butanol and the intermediate 2-butanone is a challenge throughout the cultivations and lowers 2-butanol concentrations and yields. 


\section{Materials and methods}

\section{Strains}

The Lactobacillus diolivorans strains used in this study were LMG 19667 wild type and LMG 19668+pSHM+PDO-DH(NADPH). The engineered strain LMG 19668+pSHM+PDO-DH (pduQ) possesses a overexpression of the endogenous 1,3-propanediol oxidoreductase (PDO-DH) [20]. For bioreactor cultivations, Serratia marcescens DSMZ 14187 was used.

Cells were maintained at $-80{ }^{\circ} \mathrm{C}$ in culture medium supplemented with $10 \%(\mathrm{v} / \mathrm{v})$ glycerol.

\section{2-Butanol toxicity test}

An overnight culture of $L$. diolivorans LMG 19667 in the exponential growth phase was used for inoculation of $2 \mathrm{~mL}$ MRS media with increasing concentrations of 2-butanol, ranging from 0 to $60 \mathrm{~g} / \mathrm{L}$. The initial $\mathrm{OD}_{600}$ for all cultivations was 0.1 . The inoculated cultures were incubated at $30{ }^{\circ} \mathrm{C}$ and $150 \mathrm{rpm}$ in an anaerobic jar on a rotary shaker for $72 \mathrm{~h}$. The toxicity of 2-butanol was assessed via measurement of $\mathrm{OD}_{600}$ after $72 \mathrm{~h}$ of incubation.

\section{Co-cultivation of S. marcescens and L. diolivorans}

Co-cocultivation of $S$. marcescens and $L$. diolivorans was realised in a two-step process. In the first step, a batch cultivation of $S$. marcescens was performed. Therefore, $S$. marcescens batch medium was inoculated to an $\mathrm{OD}_{600}$ of 0.2 with an overnight culture. The overnight culture of $S$. marcescens was grown on LB medium at $30^{\circ} \mathrm{C}$ and $180 \mathrm{rpm}$ on a shaker.

The co-cultivation was performed in a DASGIP ${ }^{\circledR}$ parallel bioreactor systems (Eppendorf International). For the bioreactor cultivation, in the first step, the stirrer speed and temperature were set to $400 \mathrm{rpm}$ and $30{ }^{\circ} \mathrm{C}$. The $\mathrm{pH}$ was kept constant at 7.0 via addition of $5 \mathrm{M}$ $\mathrm{NaOH}$ or $1 \mathrm{M} \mathrm{H}_{3} \mathrm{PO}_{4}$. Batch cultivations were carried out under microaerophilic conditions by gassing with $27 \mathrm{sL} / \mathrm{h}$ of air $(0.75 \mathrm{vvm})$. After approximately $19 \mathrm{~h}$, all glucose was depleted and a heat inactivation at $60{ }^{\circ} \mathrm{C}$ for $30 \mathrm{~min}$ was done and afterwards the medium was cooled again to $30^{\circ} \mathrm{C}$.

Following the cooling phase and addition of 5 times concentrated MRS batch medium to ensure proper growth conditions for L. diolivorans, the second step was initiated. In the second step, a $L$. diolivorans overnight culture was used to inoculate the MRS batch medium with an $\mathrm{OD}_{600}$ of 0.1. Additionally, for the LMG $19668+$ pSHM+PDO-DH (pduQ), erythromycin $(10 \mu \mathrm{g} /$ $\mathrm{ml}$ ) was added to the batch medium to allow stable expression of the gene. The overnight culture for both strains was done on MRS medium with a $\mathrm{pH}$ of 5.7. For the engineered strain LMG 19668+pSHM+PDO-DH (pduQ), again erythromycin $(10 \mu \mathrm{g} / \mathrm{ml})$ was added to MRS medium. The overnight culture was incubated at $30{ }^{\circ} \mathrm{C}$ and $150 \mathrm{rpm}$ for approximately $20 \mathrm{~h}$. For the bioreactor cultivation stirrer speed was set to $400 \mathrm{rpm}$ and the temperature was set to $30{ }^{\circ} \mathrm{C}$. The $\mathrm{pH}$ was kept constant at $\mathrm{pH} 5.7$ via addition of $12.5 \% \mathrm{NH}_{3}$. The bioreactor cultivation during the second step was carried out under anaerobic condition by gassing with $2 \mathrm{sL} / \mathrm{h}$ of nitrogen. For the wild-type and pduQ overexpression strain, three independent replicates were performed.

LB medium contained per litre: $10 \mathrm{~g}$ soy peptone, $5 \mathrm{~g}$ yeast extract, $5 \mathrm{~g} \mathrm{NaCl}$.

Serratia marcescens batch medium contained per litre: $33.36 \mathrm{~g}$ yeast extract, $11.39 \mathrm{~g}$ tri-sodiumcitrate $\cdot 2 \mathrm{H}_{2} \mathrm{O}$, $4 \mathrm{~g} \mathrm{NaAc}, 1 \mathrm{~g} \mathrm{NH}\left(\mathrm{HPO}_{4}\right)_{2}, 0.62 \mathrm{~g} \mathrm{MgCl}_{2} \cdot 7 \mathrm{H}_{2} \mathrm{O}, 0.11 \mathrm{~g}$ $\mathrm{MnSO}_{4} \cdot \mathrm{H}_{2} \mathrm{O}$ and $99 \mathrm{~g}$ glucose $\cdot \mathrm{H}_{2} \mathrm{O}$ [23].

MRS batch medium contained per litre: $10 \mathrm{~g}$ casein peptone, $10 \mathrm{~g}$ meat extract, $5 \mathrm{~g}$ yeast extract, $1 \mathrm{~g}$ tween $80,2 \mathrm{~g} \mathrm{~K}_{2} \mathrm{HPO}_{4}, 5 \mathrm{~g} \mathrm{NaAc}, 2.60 \mathrm{~g}$ tri-sodiumcitrate $2 \mathrm{H}_{2} 0$, $1.17 \mathrm{~g} \quad \mathrm{NH}_{4}\left(\mathrm{HPO}_{4}\right)_{2}, \quad 0.20 \mathrm{~g} \quad \mathrm{MgCl}_{2} \cdot 7 \mathrm{H}_{2} \mathrm{O}, \quad 0.05 \mathrm{~g}$ $\mathrm{MnSO}_{4} \cdot \mathrm{H}_{2} \mathrm{O}, 5 \mathrm{mg}$ vitamine $\mathrm{B} 12$ and $33 \mathrm{~g}$ glucose $\cdot \mathrm{H}_{2} 0$.

MRS medium contained per litre: $10 \mathrm{~g}$ casein peptone, $10 \mathrm{~g}$ meat extract, $5 \mathrm{~g}$ yeast extract, $1 \mathrm{~g}$ tween $80,2 \mathrm{~g}$ $\mathrm{K}_{2} \mathrm{HPO}_{4}, 5 \mathrm{~g}$ NaAc, $2.60 \mathrm{~g}$ tri-sodiumcitrate $\cdot 2 \mathrm{H}_{2} 0,1.17 \mathrm{~g}$ $\mathrm{NH}_{4}\left(\mathrm{HPO}_{4}\right)_{2}, 0.20 \mathrm{~g} \mathrm{MgCl}_{2} \cdot 7 \mathrm{H}_{2} \mathrm{O}, 0.05 \mathrm{~g} \mathrm{MnSO}_{4} \cdot \mathrm{H}_{2} \mathrm{O}$ and 22 g glucose $\cdot \mathrm{H}_{2} 0$.

\section{HPLC analysis}

The concentrations of metabolites (glucose, lactate, acetate, ethanol, meso-2,3-BTD, 2-butanone, 2-butanol) were determined by HPLC (Shimadzu, Korneuburg Austria) equipped with an Aminex HPX-87H column $(300 \times 7.8 \mathrm{~mm}$, Biorad $)$, which was operated at a temperature of $60{ }^{\circ} \mathrm{C}$ and a flow of $0.6 \mathrm{ml} / \mathrm{min}$. As a mobile phase, $4 \mathrm{mM} \mathrm{H}_{2} \mathrm{SO}_{4}$ was used. The samples and standards for HPLC analysis were prepared by mixing $900 \mu \mathrm{L}$ of sample/standard with $100 \mu \mathrm{L} 40 \mathrm{mM} \mathrm{H}_{2} \mathrm{SO}_{4}$. Subsequently, samples and standards were filtered. For the detection of metabolites, a refraction index detector (RID-10A, Shimadzu, Korneuburg Austria) was used.

\section{Abbreviations}

meso-2,3-BTD: meso-2,3-butanediol; ABE: acetone-butanol-ethanol; 2,3-BTD: 2,3-butanediol; BMC: bacterial microcompartment; 1,3-PDO: 1,3-propanediol; 3-HPA: 3-hydroxypropionaldehyde.

\section{Acknowledgements}

The authors thank Konstanze Luise Brandauer for her assistance with bioreactor cultivations.

\section{Authors' contributions}

HR designed and performed all experiments and drafted the manuscript. $\mathrm{HM}$ was involved in planning of the experiments and data interpretation. MS designed the study, was involved in planning of all experiments and data 
interpretation and finalised the manuscript. All authors read and approved the final manuscript.

\section{Funding}

The financial support by the Austrian Federal Ministry for Digital and Economic Affairs and the National Foundation for Research, Technology and Development through the Christian Doppler research Association is gratefully acknowledged. The study was furthermore financially supported by Vogelbusch $\mathrm{GmbH}$ and OMV Refining \& Marketing $\mathrm{GmbH}$.

\section{Availability of data and materials}

All data generated or analysed during this study are included in this published article.

\section{Ethics approval and consent to participate}

Not applicable.

\section{Consent for publication}

Not applicable.

\section{Competing interests}

The authors declare that they have no competing interests.

\section{Author details}

${ }^{1}$ CD Laboratory for Biotechnology of Glycerol, Muthgasse 18, 1190 Vienna, Austria. ${ }^{2}$ Department of Biotechnology, Institute of Microbiology and Microbial Biotechnology, BOKU-VIBT University of Natural Resources and Life Sciences, Vienna, Muthgasse 18, 1190 Vienna, Austria. ${ }^{3}$ ACIB GmbH, Muthgasse 11, 1190 Vienna, Austria.

Received: 30 July 2019 Accepted: 16 October 2019

Published online: 06 November 2019

\section{References}

1. Dürre P. Biobutanol: an attractive biofuel. Biotechnol J. 2007;2:1525-34

2. Chen C-T, Liao JC. Frontiers in microbial 1-butanol and isobutanol production. FEMS Microbiol Lett. 2016;363:fnw020.

3. Xue C, Zhao J, Lu C, Yang S-T, Bai F, Tang I-C. High-titer n-butanol production by clostridium acetobutylicum JB200 in fed-batch fermentation with intermittent gas stripping. Biotechnol Bioeng. 2012;109:2746-56.

4. Xu M, Zhao J, Yu L, Tang I-C, Xue C, Yang S-T. Engineering Clostridium acetobutylicum with a histidine kinase knockout for enhanced n-butanol tolerance and production. Appl Microbiol Biotechnol. 2015;99:1011-22.

5. Xue C, Zhao J, Chen L, Yang ST, Bai F. Recent advances and state-of-theart strategies in strain and process engineering for biobutanol production by Clostridium acetobutylicum. Biotechnol Adv. 2017;35:310-22.

6. Atsumi S, Hanai T, Liao JC. Non-fermentative pathways for synthesis of branched-chain higher alcohols as biofuels. Nature. 2008;451:86-9.

7. Lindlbauer KA, Marx H, Sauer M. Effect of carbon pulsing on the redox household of Lactobacillus diolivorans in order to enhance 1,3-propanediol production. N Biotechnol. 2017:34:32-9.
8. Zhang L, Guo Z, Chen J, Xu Q, Lin H, Hu K, et al. Mechanism of 2,3-butanediol stereoisomers formation in a newly isolated Serratia sp. T241. Sci Rep. 2016;6:19257.

9. Ji X-J, Huang H, Ouyang P-K. Microbial 2,3-butanediol production: a stateof-the-art review. Biotechnol Adv. 2011:29:351-64.

10. Yang T, Rao Z, Zhang X, Xu M, Xu Z, Yang S-T. Metabolic engineering strategies for acetoin and 2,3-butanediol production: advances and prospects. Crit Rev Biotechnol. 2017;37:990-1005.

11. Zhang L, Yingli S, Fed-batch IÁSÁ. Microbial production of 2, 3-butanediol by a surfactant (serrawettin)-deficient mutant of Serratia marcescens H30. J Ind Microbiol Biotechnol. 2010;37:857-62.

12. Sauer M, Russmayer H, Grabherr R, Peterbauer CK, Marx H. The efficient clade: lactic acid bacteria for industrial chemical production. Trends Biotechnol. 2017:35:756-69.

13. Pflügl S, Marx H, Mattanovich D, Sauer M. 1,3-Propanediol production from glycerol with Lactobacillus diolivorans. Bioresour Technol. 2012;119:133-40.

14. Patakova P, Kolek J, Sedlar K, Koscova P, Branska B, Kupkova K, et al. Comparative analysis of high butanol tolerance and production in clostridia. Biotechnol Adv. 2018:36:721-38.

15. Knoshaug EP, Zhang M. Butanol tolerance in a selection of microorganisms. Appl Biochem Biotechnol. 2009;153:13-20.

16. Branduardi P, Longo V, Berterame NM, Rossi G, Porro D. A novel pathway to produce butanol and isobutanol in Saccharomyces cerevisiae. Biotechnol Biofuels. 2013;6:68.

17. Speranza G, Corti S, Fontana G, Manitto P, Galli A, Scarpellini M, et al. Conversion of meso-2,3-butanediol into 2-butanol by Lactobacilli. Stereochemical and enzymatic aspects. J Agric Food Chem. 1997;45:3476-80.

18. Rao B, Zhang LY, Sun J, Su G, Wei D, Chu J, et al. Characterization and regulation of the 2,3-butanediol pathway in Serratia marcescens. Appl Microbiol Biotechnol. 2012;93:2147-59.

19. Lindlbauer KA, Marx H, Sauer M. 3-Hydroxypropionaldehyde production from crude glycerol by Lactobacillus diolivorans with enhanced glycerol uptake. Biotechnol Biofuels. 2017;10:295.

20. Pflügl S, Marx H, Mattanovich D, Sauer M. Genetic engineering of Lactobacillus diolivorans. FEMS Microbiol Lett. 2013:344:152-8.

21. Ghiaci P, Lameiras F, Norbeck J, Larsson C. Production of 2-butanol through meso-2,3-butanediol consumption in lactic acid bacteria. FEMS Microbiol Lett. 2014;360:70-5

22. Branduardi P, de Ferra F, Longo V, Porro D. Microbial n-butanol production from Clostridia to non-Clostridial hosts. Eng Life Sci. 2014;14:16-26.

23. Li J, Zhao JB, Zhao M, Yang YL, Jiang WH, Yang S. Screening and characterization of butanol-tolerant micro-organisms. Lett Appl Microbiol. 2010;50:373-9.

24. Ghiaci P, Norbeck J, Larsson C. 2-Butanol and butanone production in Saccharomyces cerevisiae through combination of a B12 dependent dehydratase and a secondary alcohol dehydrogenase using a TEV-based expression system. PLoS ONE. 2014;9:e102774

\section{Publisher's Note}

Springer Nature remains neutral with regard to jurisdictional claims in published maps and institutional affiliations.

Ready to submit your research? Choose BMC and benefit from

- fast, convenient online submission

- thorough peer review by experienced researchers in your field

- rapid publication on acceptance

- support for research data, including large and complex data types

- gold Open Access which fosters wider collaboration and increased citations

- maximum visibility for your research: over 100M website views per year

At BMC, research is always in progress.

Learn more biomedcentral.com/submissions 